\title{
Tubulações em sistemas de recalque
}

\author{
Methodology for determination of the economic diameter tube in impulsion line system
}

\author{
Adroaldo Dias Robaina ${ }^{1}$ Marcelo Calgaro² Márcia Xavier Peiter $^{3}$
}

\section{RESUMO}

$O$ presente trabalho teve como objetivo o desenvolvimento de uma metodologia para a determinação do diâmetro mais econômico de uma tubulação de recalque de sistema de bombeamento de água para a irrigação. $O$ trabalho apresenta como principais diferenças de outros procedimentos semelhantes à utilização de uma função do preço da tubulação por unidade de peso e por metro linear, uma função do preço do conjunto motobomba por unidade de potência, e a utilização da fórmula universal de perda de carga, que leva em conta a natureza da parede da tubulação e o regime de escoamento a que se encontra submetido. A expressão final para a obtenção do diâmetro econômico permitiu relacionar os diversos elementos intervenientes na solução do problema de uma maneira bastante simples, tendo a necessidade de um processo iterativo, mas bastante rápido quanto à convergência para a solução do problema.

Palavras-chave: diâmetro ótimo, recalque de água, irrigação.

\section{ABSTRACT}

The objective of the present research was to develop a methodology to determine efficiency tube diameter in impulsion line of pump system for irrigation. This work had some differences compared with similar procedures. The utilization of a function of tubes price for weight unit and for linear meter, also a price function of pump for potential unit and utilization of the universal equation of lost head depend on tube wall with a friction regimen. A final equation to obtain an efficient diameter allowed to stablish relationship among different elements to resolve the problem in a simple way, being necessary an interactive process, but faster as possible to reach a solution for a problem.

Key words: optimal diameter, impulsion line, irrigation.

\section{INTRODUÇÃO}

O diâmetro das tubulações de recalque dos sistemas de bombeamento é um problema hidraulicamente indeterminado. Segundo ROBAINA (1997), o recalque com velocidades baixas, resulta em diâmetros relativamente grandes, implicando um custo elevado para as tubulações e em menores gastos com energia consumida e com o conjunto motobomba, pelo fato de resultar em menores alturas manométricas. Por outro lado, velocidades altas possibilitam o uso de diâmetros menores, de custo menor, mas que resultam em perdas de carga mais elevadas e, conseqüentemente, exigem maior gasto com conjunto motobomba e com o consumo de energia. Segundo NEVES (1974), o problema da escolha do diâmetro da tubulação de recalque apresenta infinitas soluções sob o ponto de vista hidráulico e a indeterminação é levantada admitindose uma outra condição, chamada de condição de mínimo custo total do sistema de bombeamento (custo da tubulação, bomba e motor) e operacional (energia consumida). Existem vários trabalhos semelhantes como o proposto por TELES (1967), CARNEIRO (1976) MAGNANI (1977), NEVES (1974) e RICHTER (1983), sendo que a maioria dos técnicos utiliza uma fórmula prática, como a de Hazen-Williams, para o cálculo da perda de carga, ou outras fórmulas que não levam em conta o fator de recuperação do capital investido na aquisição da tubulação e na motobomba ou não

${ }^{1}$ Engenheiro Agrônomo, Professor Titular, Depto. de Engenharia Rural, Centro de Ciências Rurais (CCR), Universidade Federal de Santa Maria (UFSM), 97059-900, Santa Maria, RS. Tel.(55) 220-8261. Email: arobaina @ccr.ufsm.br

${ }^{2}$ Engenheiro Agrônomo, Doutorando do Programa de Pós-graduação em Engenharia Agrícola, CCR, UFSM. Santa Maria, RS. Email: calgaro@mail.ufsm.br;Autor para correspondência.

${ }^{3}$ Engenheiro Agrônomo, Professora do Curso de Engenharia Agrícola, Universidade Regional Integrada do Alto Uruguai e das Missões (URI), Campus de Santiago, RS. E-mail: mpeiter@urisantiago.br. 
expressam o custo da tubulação em função do peso por metro linear.

O presente trabalho teve como objetivo apresentar uma metodologia para o cálculo do diâmetro ótimo de tubulações de recalque de sistemas de bombeamento de água para irrigação, com base nos custos das tubulações e do conjunto motobomba e no custo da energia consumida.

A metodologia proposta utiliza a fórmula Universal para a determinação da perda de carga, que tem como vantagem a possibilidade de ser utilizada para qualquer tipo de tubo e regime de escoamento.

\section{MATERIAL E MÉTODOS}

Neste trabalho, foram utilizados os preços de tubos de aço soldado, comercializados na região de Santa Maria-RS, no período de janeiro a abril de 2003. No entanto, essa metodologia poderá ser adaptada para outros tipos de tubulações, bastando determinar o preço dos diferentes tipos de tubos.

A metodologia usada para a determinação do diâmetro econômico de uma tubulação de recalque de um sistema de bombeamento baseia-se na otimização do custo total anual do sistema de bombeamento. Esse custo pode ser expresso por:

$$
C_{T}=C_{T u}+C_{M B}+C_{O p}
$$

onde $C_{T}$ é o custo total anual do sistema, $C_{T u}$ é o custo anual da tubulação de recalque (amortização e juros), $C_{M B}$ é o custo anual do conjunto moto-bomba (amortização e juros) e $C_{O p}$ é o custo anual de operação (energia consumida). Derivando a expressão (1) em relação ao diâmetro, obtém-se:

$$
\frac{\partial C_{T}}{\partial D}=\frac{\partial C_{T u}}{\partial D}+\frac{\partial C_{M B}}{\partial D}+\frac{\partial C_{O p}}{\partial D}
$$

Estabelecendo-se a condição de custo total mínimo do sistema, que é $\frac{\partial C_{T}}{\partial D}=0$ sujeita à condição $\frac{\partial^{2} C_{T}}{\partial D^{2}}>0$

Sendo o peso dos tubos estimados por ,$P_{T u}=\gamma_{m} . \pi$.D.e.L o custo anual da tubulação de recalque pode ser expresso por:

$$
C_{T u}=K_{1} \cdot \gamma_{m} . \pi \cdot \text { D.e.L.FRC }
$$

na qual $\mathrm{K} 1$ é o preço da tubulação por unidade de peso e por metro de tubo (R\$/kgf.m), $\gamma_{\mathrm{m}}$ é o peso específico do material da tubulação $\left(\mathrm{kg} f \cdot \mathrm{m}^{-3}\right), \mathrm{D}$ o diâmetro do tubo (m), e é a espessura do tubo (m), L o comprimento total da tubulação $(m)$ e $F R C$ é o fator de recuperação do capital empregado na aquisição da tubulação.
O custo anual do conjunto motobomba pode ser expresso por:

$$
C_{M B}=K_{2} \cdot 0,736 . F R C . P
$$

onde $K_{2}$ é o preço do conjunto motobomba por unidade de potência $(R \$ / C v)$, $\mathrm{P}$ é a potência do conjunto motobomba $(C v)$ e 0,736 é o fator de conversão de $C v$ para $k W$.

O custo anual de operação (energia consumida) do sistema de bombeamento pode ser expresso por:

$$
C_{O p}=K_{3} \cdot 0,736 \cdot N h \cdot P
$$

na qual $K_{3}$ é o preço por unidade de energia consumida $(R \$ / k W h)$, considerando-se acionamento por motor elétrico, e Nh é o número de horas de operação por ano.

A potência de um sistema de bombeamento, utilizando a fórmula universal de perda de carga, pode ser expressa por:

$$
P=\frac{\gamma \cdot Q \cdot H g}{75 \cdot R g}+\frac{0,0826 \cdot \gamma \cdot f \cdot Q^{3} \cdot L}{75 \cdot R g \cdot D^{5}}
$$

na $\gamma$ é peso específico do líquido bombeado $\left(\mathrm{kgf.m}^{-3}\right)$, $Q$ a vazão $\left(\mathrm{m}^{3} \cdot \mathrm{s}^{-1}\right), R g o$ rendimento do conjunto motobomba (decimal) e f o fator de perda de carga, um número puro, expresso em função da rugosidade relativa da tubulação ( $\varepsilon . \mathrm{D}^{-1}$ ) e do Número de Reynolds (NR), sendo e a rugosidade absoluta $(\mathrm{m})$. Derivando-se a equação 3, tem-se $\frac{\partial C_{T}}{\partial D}=K_{1} \cdot \gamma_{m} \cdot \pi \cdot e \cdot L . F R C$ e sendo $k_{4}=k_{1} \cdot \gamma_{m} \pi . e . L . F R C$ obtém-se:

$$
\frac{\partial C_{T}}{\partial D}=K_{4}
$$

Derivando-se a equação 4, tem-se:

$$
\frac{\partial C_{M B}}{\partial D}=K_{2} \cdot 0,736 \cdot F R C \cdot \frac{\partial P}{\partial D}
$$

e sendo $k_{5}=k_{2} \cdot 0,736 . F R C$ obtém-se:

$$
\frac{\partial C_{M B}}{\partial D}=K_{5} \cdot \frac{\partial P}{\partial D}
$$

Derivando-se a equação 5, tem-se:

$$
\frac{\partial C_{O p}}{\partial D}=K_{3} \cdot 0,736 \cdot N h \cdot \frac{\partial P}{\partial D}
$$

e sendo $k_{6}=k_{3} .0,736 . N h$, obtém-se:

$$
\frac{\partial C_{O p}}{\partial D}=K_{6} \cdot \frac{\partial P}{\partial D}
$$

Derivando-se a equação 6 , tem-se:

$$
\frac{\partial P}{\partial D}=\frac{-5 \cdot \gamma}{75 \cdot R g} \cdot \frac{0,0826 \cdot f \cdot Q^{3} \cdot L}{D^{6}}
$$

Ciência Rural, v.34, n.4, jul-ago, 2004. 
e sendo $J=\frac{0,0826 . f . Q^{2}}{D^{5}} \quad$ obtém-se:

$$
\frac{\partial P}{\partial D}=\frac{-5 \cdot \gamma}{75 \cdot R g} \cdot \frac{J \cdot Q \cdot L}{D}
$$

Substituindo-se o valor de $\frac{\partial P}{\partial D}$ da equação 10 , nas equações 8 e 9 e colocando as equações 7,8 e 9 na expressão (2), obtém-se:

$k_{1} \gamma_{m} \pi e F R C-\left(k_{2} 0,736 F R C+k_{3} 0,736 N h\right) \frac{5 \gamma J Q}{75 \operatorname{Rg} D}=0_{(11)}$

Explicitando a perda de carga unitária $\mathbf{J}\left(\mathrm{m} . \mathrm{m}^{-1}\right)$, a partir da equação 11 , pode-se obter o seu valor pela seguinte expressão:

$$
J=\frac{15 R g k_{1} \gamma_{m} \pi e F R C}{0,736 \gamma\left(k_{2} \cdot F R C+k_{3} \cdot N h\right)} \frac{D}{Q}
$$

Segundo QUINTELA (1981), com base na vazão $\mathrm{Q}\left(\mathrm{m}^{3} \cdot \mathrm{s}^{-1}\right)$, na perda de carga unitária $\mathrm{J}\left(\mathrm{m} \cdot \mathrm{m}^{-1}\right)$, na rugosidade absoluta e $(\mathrm{m})$ e na viscosidade cinemática $v$ do fluído bombeado $\left(\mathrm{m}^{2} . \mathrm{s}^{-1}\right)$, o valor do diâmetro $\mathrm{D}(\mathrm{m})$ pode ser obtido iterativamente por:

$$
D_{i+1}=\left(\frac{2 Q}{\pi \sqrt{2 g J}}\right)^{2 / 5} \frac{1}{\log 2 / 5\left(\frac{\varepsilon}{3,7 D_{i}}+\frac{2,51 v}{D_{n} \sqrt{2 g J D_{i}}}\right)}
$$

Fazendo-se $k_{7}=\frac{15 R g k_{1} \gamma_{m} \pi e F R C}{0,736 \gamma\left(k_{2} \cdot F R C+k_{3} \cdot N h\right)}$ a equação 12 se transforma em $\quad J=k_{7} \frac{D}{Q} \quad$ valor uti lizado na equação 13.

Após manipulações algébricas e simplificações obtem-se:

$$
D_{i+1}=\frac{0,5238 Q^{1 / 2}}{\left[k_{7} \cdot \log ^{2}\left(\frac{0,27 \cdot \varepsilon}{D_{i}}+\frac{0,57 \cdot v \cdot Q^{1 / 2} / k_{7}^{1 / 2}}{D_{i}^{2}}\right)\right]^{1 / 6}}
$$

\section{RESULTADOS E DISCUSSÃO}

A equação 14 possibilita determinar o diâmetro econômico de uma tubulação de recalque de sistemas de bombeamento de água. A Tabela 1 apresenta o peso dos tubos de aço, fabricados com duas espessuras diferentes (2,00 e 2,65 mm), e os preços praticados por peso do tubo. Nessa tabela, pode-se ver a comparação entre os pesos fornecidos pelo fabricante e os estimados neste trabalho. Os valores mostrados na tabela permitem observar que a função $\left(P_{T u}=\gamma_{m} . \pi\right.$.D.e.L $)$ utilizada para estimar o peso do tubo se ajusta bem aos valores reais.

Adotando-se como preço médio o valor de $\mathrm{R} \$ 2,55$, obtido pela média dos valores das duas espessuras consideradas $(\mathrm{R} \$ 2,58$ para $\mathrm{e}=2,00 \mathrm{~mm} \mathrm{e}$ $\mathrm{R} \$ 2,52$ para $\mathrm{e}=2,65 \mathrm{~mm})$ e considerando um acréscimo de $7 \%$ atribuído a IPI, pode-se estimar um valor de $\mathrm{R} \$ 2,73$ para o preço por unidade de

\begin{tabular}{|c|c|c|c|c|c|c|}
\hline \multirow{3}{*}{$\mathrm{D}(\mathrm{mm})$} & \multicolumn{4}{|c|}{ Pesos dos Tubos de aço (kgf.m $\left.{ }^{-1}\right)$} & \multicolumn{2}{|c|}{ Preço (R $\$ / k g f)$} \\
\hline & \multicolumn{2}{|c|}{$\mathrm{e}=2,00 \mathrm{~mm}$} & \multicolumn{2}{|l|}{$\mathrm{e}=2,65 \mathrm{~mm}$} & \multirow{2}{*}{$\begin{array}{l}\mathrm{e}=2,00 \mathrm{~mm} \\
\text { Fabricante }\end{array}$} & \multirow{2}{*}{$\begin{array}{l}\mathrm{e}=2,65 \mathrm{~mm} \\
\text { Fabricante }\end{array}$} \\
\hline & Fabricante & Estimado & Fabricante & Estimado & & \\
\hline 150 & 8,00 & 7,92 & 10,50 & 10,49 & 3,00 & 2,95 \\
\hline 175 & 9,00 & 9,24 & 12,00 & 12,24 & 2,89 & 2,83 \\
\hline 200 & 10,00 & 10,56 & 14,00 & 13,99 & 2,65 & 2,61 \\
\hline 250 & 13,00 & 13,19 & 17,50 & 17,48 & 2,58 & 2,51 \\
\hline 300 & 15,00 & 15,83 & 21,00 & 20,98 & 2,53 & 2,52 \\
\hline 320 & 16,00 & 16,89 & 22,00 & 22,38 & 2,50 & 2,50 \\
\hline 350 & 17,50 & 18,47 & 24,00 & 24,48 & 2,57 & 2,46 \\
\hline 380 & 19,20 & 20,06 & 26,00 & 26,57 & 2,50 & 2,47 \\
\hline 400 & 20,00 & 21,11 & 28,00 & 27,97 & 2,50 & 2,43 \\
\hline 450 & 23,00 & 23,75 & 31,00 & 31,47 & 2,52 & 2,42 \\
\hline 480 & 24,00 & 25,33 & 33,00 & 33,57 & 2,46 & 2,42 \\
\hline 500 & 25,00 & 26,39 & 35,00 & 34,97 & 2,48 & 2,40 \\
\hline 550 & 28,00 & 29,03 & 38,00 & 38,46 & 2,39 & 2,37 \\
\hline 600 & $\mathrm{nd}^{*}$ & 31,67 & 42,00 & 41,96 & nd & 2,38 \\
\hline MÉDIA & & & & & 2,58 & 2,52 \\
\hline
\end{tabular}
peso dos tubos de aço.

Tabela 1 - Pesos fornecidos pelo fabricante e estimados dos tubos nas espessuras de 2,00 e 2,65mm e preços praticados por unidade de peso de tubos de aço, para as duas espessuras respectivamente $(R \$ / \mathrm{kg} f)$.

* não disponível. 
Na Tabela 2, pode-se observar a variação de preços dos conjuntos motobombas elétricos e a variação dos preços por unidade de potência, não considerando o preço dos equipamentos de proteção e manobra. Pode-se verificar nessa tabela que o preço por unidade de potência é, aproximadamente, constante, apresentando o valor médio de $\mathrm{R} \$ 135,60$ por unidade de potência.

A Tabela 3 mostra os resultados para diversas vazões em função de diversos elementos que interferem no cálculo do diâmetro mais econômico de uma tubulação de recalque de sistemas de bombeamento de água. Os valores utilizados para a simulação foram: $K_{1}=R \$ 2,73$, $\mathrm{K}_{2}=\mathrm{R} \$ 135,60, \mathrm{Nh}=2000 \mathrm{~h}, \mathrm{e}=2 \mathrm{~mm}, \mathrm{v}=0,000001 \mathrm{~m}^{2} \cdot \mathrm{s}^{-}$ ${ }^{1}, \gamma_{\mathrm{m}}=8400 \mathrm{kgf} \cdot \mathrm{m}^{-3}, \mathrm{e}=0,25 \mathrm{~mm}$ e $\mathrm{Rg}=0,70$.

A tabela 3 apresenta também a influência do FRC na determinação do diâmetro de uma tubulação de recalque e da energia consumida para um mesmo FRC, mostrando que a velocidade da água variou de 0,87 a $1,24 \mathrm{~m} . \mathrm{s}^{-1}$, para uma ampla gama de vazões simuladas.

\section{CONCLUSÕES}

A metodologia proposta permite obter o diâmetro econômico da tubulação de um sistema de bombeamento de uma maneira simples e rápida, apesar de utilizar um processo iterativo para a solução do problema.

Tabela 2 - Preços de conjuntos motobomba e preços por unidade de potência, considerando acionamento com motores elétricos.

\begin{tabular}{lll}
\hline Potência $c v$ & $\begin{array}{l}\text { Conjunto } \\
\text { motobomba }\end{array}$ & Unitário \\
\hline & $R \$$ & $R \$ / c v$ \\
15 & 2074,00 & 138,27 \\
25 & 3422,00 & 136,88 \\
30 & 4092,00 & 136,40 \\
50 & 6750,00 & 135,00 \\
75 & 10044,00 & 133,92 \\
100 & 13315,00 & 133,15 \\
MÉDIA & & 135,60 \\
\hline
\end{tabular}

Tabela 3 - Diâmetro $(\mathrm{mm})$ econômico e respectivas velocidades $\left(m . s^{-1}\right)$ para diferentes vazões simulando variações do fator de recuperação de capital e preço da energia consumida.

\begin{tabular}{|c|c|c|c|c|c|c|c|}
\hline & Valor & & $g n$ & e $K_{3}=$ & Valore & $\begin{array}{l}K_{3}-R \$ / k \\
=15 \%\end{array}$ & $W h$ e FKC \\
\hline 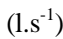 & 10 & & 15 & 20 &, 15 &, 20 & 0,25 \\
\hline & 0, & & & 91 & 1761 & & 1930,87 \\
\hline & 2620 , & 6 & & 51 & 2461 & 2570,96 & 266 \\
\hline & 3180, & 9 & 1,06 & 2861,16 & 2991,06 & 3120,98 & 3230,91 \\
\hline 00 & 3650, & 3 & 1 , & 3291,17 & 7431,08 & 3590,98 & 3710,92 \\
\hline 0 & 4440 , & 7 & 1 , & 3991,19 & 4171,09 & 4361,00 & 4520,93 \\
\hline 0 & 5100, & 9 & 1 , & 591,20 & 4791,10 & 5011,01 & 5190,94 \\
\hline 50 & $\begin{array}{lll}568 & 0,98\end{array}$ & 4 & 1,1 & 5111,21 & 5341,1 & 5581,02 & 5780,95 \\
\hline 00 & 6200,9 & 583 & 1,12 & 5881,22 & 5831 , & 6091,02 & 6310,95 \\
\hline 0 & 7941,0 & 746 & 1,1 & 7141,24 & 7461 , & 7791,04 & 8070,97 \\
\hline
\end{tabular}

\section{REFERÊNCIASBIBLIOGRÁFICAS}

CARNEIRO, B.P. Otimização dos custos de uma instalação de recalque. Revista DAE, n.104, p.68-80, 1976.

MAGNANI, J.R. Determinação do diâmetro econômico de adutoras e emissários por recalque. Revista DAE, n.113, p.75-87, 1977.

NEVES, E.T. Curso de hidráulica. Porto Alegre : Globo, 1974. 577 p.

QUINTELA, A. de C. Leis de resistência dos escoamentos uniformes. In: Hidráulica. Lisboa : Fundação Calouste Gulbenkian, 1981. Cap.7, p.123164 .

RICHTER, C.A. Diâmetro econômico das canalizações de recalque (adaptação e atualização da fórmula de Koch e Vibert). Revista DAE, n.133, p.33-34, 1983 .

ROBAINA, A.D. Seleção de bombas para a irrigação. Lavoura Arrozeira, v.50, n.432, p.12-16, 1997.

TELES, E.G. Novo processo para a determinação do diâmetro mais econômico para adutora de recalque. Revista DAE, n.65, p.18-24, 1967. 